\title{
La conduite automatique de la centrale de la Rance
}

\author{
par Alain Charen \\ $\mathrm{EDF}$ \\ Directeur Groupe Mixte d'Exploitation Bretagne
}

La conduite de la centrale a pour objectif d'utiliser au mieux l'énergie des marées dans l'estuaire de La Rance.

Le bassin, créé par la centrale, s'étend jusqu'à l'écluse du Châtetier, près de Dinan, sur une vingtaine de kilomètres. La superficie du plan deau à la marée la plus forte est de 22 $\mathrm{km}^{2}$.

Le volume d'eau déplacé par les grandes marées est de I $84 \mathrm{Mm}^{3}$ pour une variation maximate du niveau de $13,5 \mathrm{~m}$.

\section{I $\square$ LES CYCIES DE FONCTIONNEMENT}

L'utilisation la plus simple conduit à réaliser des remplissages et des vidages successifs du bassin.

\subsection{Simple effet (fig 1).}

- A marée montante, on remplit le bassin par les vannes. $A$ pleine mer, on ferme les vannes et on attend pour vider te bassin par les turbines, que la mer en se retirant ait créć unc hautcur de chute suffisante.

- C'est l'cxploitation la plus simple qui étail réalisée autefois dans les moulins à maréc.

- On peut améliorer ce cycle en utilisant l'énergie disponible sur le réseau aux heures creuses pour ponper l'cat do la mer afin de surélever le niveau du bassin et d'augmenter le volume turbinable.

\subsection{Double effet}

- C'est la combinaison des deux cycles précédents. L'énergie est produite à la fois lors du remplissage ef lors du vidage du bassin.

w.- Parmi les nombreux cycles dont dispose l'exploitant, it choisira celui yui convient le mieux, selon l'amplitude de la maréc et la valeur de l'énergie aux diverses heures de la joumée.

- Grâcc au fonctionnement à double effet et au pompage, if est donc possible d'obtenir une explaitation "sur mesure ", fonction de l'heure et de l'amplitude de la marée.

\section{I LE PROCESSUS À COMMANDER}

\section{1 les vannes}

Elles son all nombre de $6: 15 \mathrm{~m}$ cie largeur $10 \mathrm{~m}$ de hatteur.

Elles sont mancruvables hor's charge : lorsque les niveaux mer et bassin sont equilibrés, elles sont mancuvrées pour être, soit totalement ouvertes, soit totalement fermées.

\section{2 les groupes}

Les groupes sont du type "bulbe amont "; ils rasscmblent dars une même coque mélallique immergée dans un conduit hydrauligue:

- une turbine Kaplan horizontale à pales et distributeur mobilc.

Diamètre de la roue : $5,35 \mathrm{~m}$

Nombre de pales : $4(-5$ à $=35$ degrés $)$

Nombre d'aubes directrices

du distributeur : 24

Vitesse normale : $93,75 \mathrm{tr} / \mathrm{mn}$

Débit maximum : $275 \mathrm{~m}^{3} / \mathrm{s}$.

- Un alternateur synchrone de $10 \mathrm{MW}$ à excitation statique fonctionnant dans l'air surpressé à 2 bars absolus sous la tension de $3500 \mathrm{~V}$.

Les groupes peuvent fonctionner indifferemment en pompe ou en turbine, leur sens de rotation étant déterminé par le sens d'écoulement de l'cau.

L'unité d'exploitation est constituée par un cnsemble de 4 groupes; ceux-ci fonctionnent simultanément el disposent en commun d'un certain nombre d'organes annexes notamment pour le réglage des turbines et l'excitation des alternateurs.

Chaque ensemble débite sur l'un des deux primaires du transformateur de " bloc" correspondant.

Il existe donc 6 ensembles et 3 blocs. ..... Auxiliaires : 
I.cs services auxiliajres ahternatifs sont alimentés à partij de deux transformateurs $63 / 5,5 \mathrm{kV}$ de 5 MVA raccordés en dérivation sur un câble reliant Dinard ì St-Malo.

Ln réseau $5,5 \mathrm{kV}$ distribue l'énergie à une série de 8 postes de transformation $5500 / 380$ Volts.

Deux groupes diesels de $600 \mathrm{kVA}$ à démarage automatiqse assurent l'alimentation des uuxiliajes essentiels en cas de perte de ta tension $63 \mathrm{kV}$.

\section{L'ORGANISATION DE LA CONDUITE}

Let réalisation des cycles de fonctionnement se fait en đ̇eux phases.

\subsection{La programmation}

La programmation tient compte :

— du cycle des marées dont la connaissance est totalement prévisible à longue échéance,

- de la disponibilité prévisionnelte des matériels de la centrate : groupes et vannes. La maintenance de la centrale est liée au cycle des marées de vive eau et de morte eat, ce qui permet de prévoir longtemps à l'avance la période d'arrêt pour entretien des machines.

- de la prévision hebdomadrure du coût de l’énergic électrique dans le cadre de la planification nationale.

- des contraintes extcmes à la centrale, notamment sur les niveaux à ne pas dépasser dans le bassin.

A partir de trutes ces données, le code de calcul appelé AGRA (Algorithme de Gestion de La Rance) propose le progranme de fonctionnement détaillé (au pas de $10 \mathrm{mn}$ ) de la centrale après avoir optimisé le fonctionnemert de l'ensemble : bassin - usine. L'optimisation est faite sur le critère de ta recette exprimée en francs.

Le programme ainsi obtentr est transmis à l'usine (figure 2).

\section{2 Le suivi du programme}

Lec programme à exícuter se présente sous la forme d'une liste aut donne au pas cte $10 \mathrm{mn}$ :

- les ordres à émettre vers les groupes et les vannes,

- les niveaux de la mer et du bassin.

De 1968 is l'automatisttion de la condute, le programme de fonctionnement hebdomadaire est en mémoire dans le programmateur (calculateur PDP 8) et est exécuté à partir de la date et de l'heure du moment.

De 1966 à 1987, un service de quart existe en salte de conmande. Il a pour fonction de surveiller be déroulement du programme et il intervient dès lors qu'il y a une mavaise exécution d'ordre ou une dérive du niveata du bassin. II doit également corriger l'exécution du programme si les conditions présentes ne sont pas celles qui onl servi à l'élaboration du programme : indisponibilité fortuite d'une machine, contrainte sur les réseaux électriques.

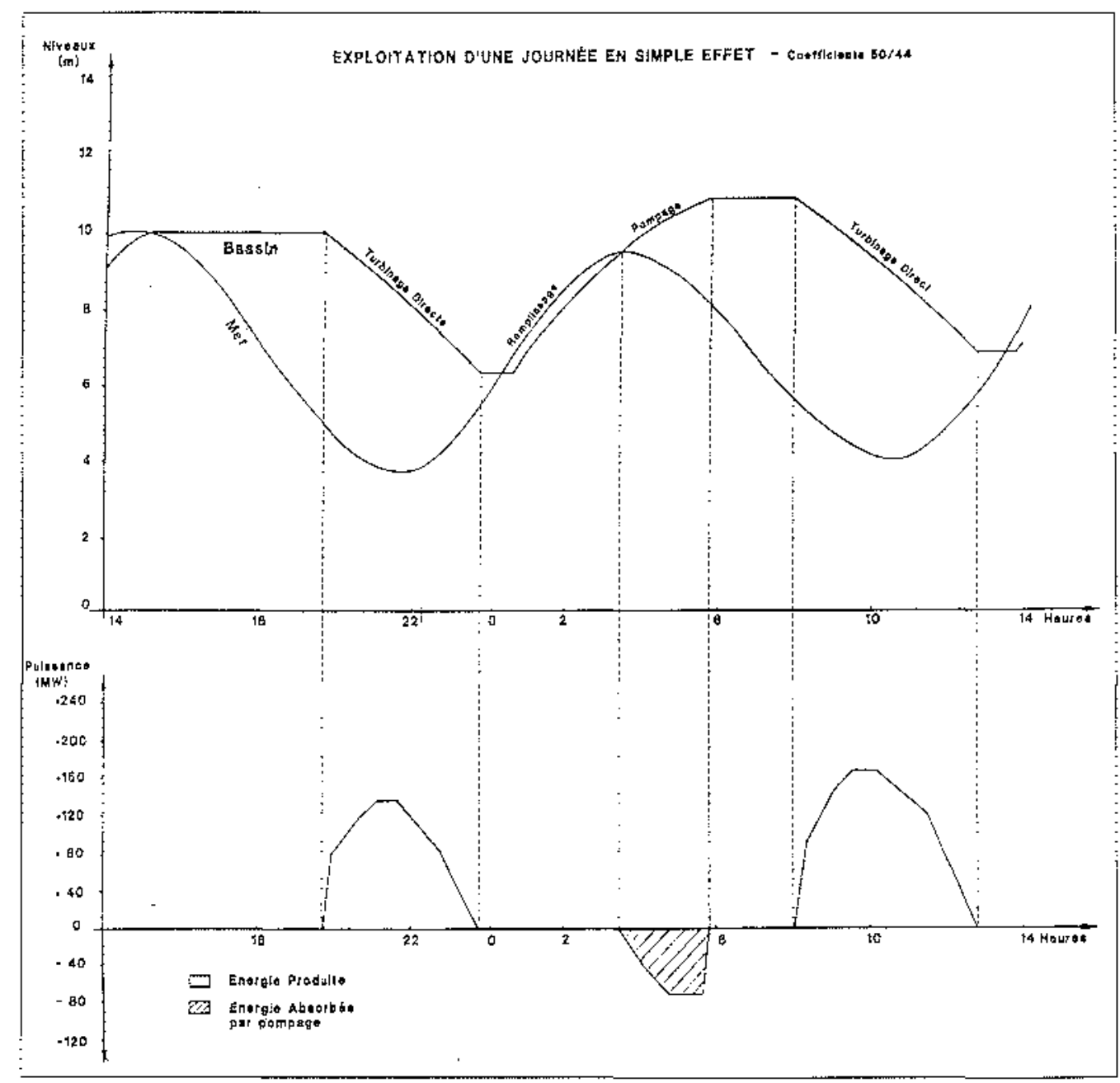

1. Exploitation d'une journée en simple effet - coefficients 50)/44. 


\section{L'AUTOMATISATION DE IA CONDLI'T}

\subsection{Les raisons du changement}

Le programmateur (PD 8) arrivait en fin de vic au début des années 1980 et il étalit devenu difficite d'asstuer la maintcnance car les pièces de rechangè ne pouvaient plus être approvisionnées. On a donc déciłé de rénover cel équipe. ment et à cette occasion, on a envisagé la conduite entièrement automatiqute de la centrate. Cette démarche s'inscrivait dans la recherche normale de la productivité par suppression du service de quart, comme cela s'est fait dans les autres centrales hydrótectriques d'EDF

\section{* 4.2 Les principales fonctions à assurer}

Ce sont celles d'un atomate de $2^{\text {ran: }}$;ang, is satwoir : ...... la réception et la validation du programme de fonctionnement issu de AGRA,

- La conduite, en temps réel, de la centrale par exécution du progranme et prise en compte des f́vénements récents et non intégrés dans le programme,

-.m- la consignation de tous les événements de la centrale, à savoir 2277 entrées TOR (tout ou rien), 207 sorties TOR, 124 entrées analogiques,

- le traitement des données en vue de leur suivi, l'archivage, le tracé des cuurbes,

- le dialogue homme-machine.

\subsection{Les principales caractéristiques de f'automatisation}

L'automatisation a nécessité la résolution de nombreux problèmes spécifiques à La Rance et dont les principaux sont citćs ci-après

- le logiciel AGRA a dû être révisé de mantière à mieux motéliser l'estuaire de La Rance at les caractéristiques des machines.

- le nombre d'entrées-sorties est très important, comparé à ce qu'on renconere habituellement dans unc contrale hydročlectrique.

- l'aménagement de La Rance n'a pas de position de repos. Sous aucun prétexte la centrale ne peut s'arrêter. La maréc ne s'arrête pas. Il faut reconstiuer une marée dans l'estuaire et la centrale est pratiquement toujours en situation dynamique. - les distunces dans la centrate sont importantes : $390 \mathrm{~m}$ de longueur pour la salle des machines. Les équipements sont complexcs: groupes regroupés par ensembles de 4,2 ensembles pour un transformateur principal.

- le niveat de la mer est influencé par la pression atmosphérique et le njveut réel est rarement identique au niveau prévu par les tableaux des marées. Il faut tenir compte de ce phénomène en temps récl (fie 3).

- le suivi de la trajectoire du niveau du bassin nécessite la mise en place d'une régutation de niveau, ef complément des ordres issus du programme. Cette régulation, appelée micro-rattrapage doit compenser les eneurs de position des groupes.

- Ie programme de fonctionnenem peu être rectitié localement afir de prendre en comple des evénements ou contraintes non pris en compte lors de la prévision.

\subsection{Les choix techniques retenus}

Compte tenu de la taille de l'usine et du coût du câblage, une struciure dfécentralisée a été retenue.

Les infomations et commancles des groupes sont câblées au niveau d'un ensemble (4 groupes) at traitées par un équipement de conduite d'ensemble (ECE). Il en est de même pour jes vannes, l'équipement de conduite des vannes assurrant de plus les informations en provenance des auxilieires.

Le relayage du premier rang a été conservé ; il assure pour 4 groupes (un ensemble) :

- la protection.

- le démarrage el l'arrêt.

-- le réglage

En ce qui concene les vannes, l'zutomate de premier rang cst propre ì chaque vanne. Les équipements de conduite d'ensemble sont situés en salle des machines : ce sont des automates programmab)es (SMC 600).

Par homogénétté, l'équipenentit de conduite de vanne est un automate programmable de même type situé en salle" calculatcur".

Les uraitements communs, tes fonctions de calculs importants son effectućs dans un calculateur central ou Equipement de Conduite Lsine (ECU) relié aux automates par des liaisons séries (CLIJ2).

\begin{tabular}{|c|c|c|c|c|c|c|c|c|c|c|c|c|c|c|c|c|}
\hline \multirow{2}{*}{; } & $\operatorname{cov} s$ & $\begin{array}{l}\text { Cork } \\
\text { BARR }\end{array}$ & COF & ChUTE & vou & \multicolumn{2}{|c|}{$\begin{array}{l}\text { YANWES } \\
\end{array}$} & \multicolumn{3}{|c|}{ GROUPES } & $\begin{array}{c}\text { PULSS } \\
\text { ins }\end{array}$ & Wuin & Rowim & PALES & cour & $\begin{array}{l}\text { Xasy } \\
\text { ongro }\end{array}$ \\
\hline & $M$ & $M$ & ant & a & XM & in & $M / S$ & צ: & is & & $k w$ & MW & 5 & WOERES & $\mathbf{P}$ & Mu \\
\hline $0 \mathrm{H} D$ & 8.14 & B.36 & 8.79 & -0.49 & 77323 & & -2761 & 22 & of & .2241 & $\theta$ & 0 & 0 & 15.00 & 245 & 200 \\
\hline OH 10 & 8.34 & 6.49 & 8.97 & .0 .48 & $B 032 x$ & & -2740 & 22 & Ot & -2192 & 0 & 0 & 0 & 15.00 & 245 & 220 \\
\hline $0 \mathrm{H} 20$ & 8.53 & 8.67 & 9.12 & -0.45 & 83285 & & -2676 & 22 & ol & .2114 & 0 & 0 & 0 & 15.00 & 245 & 220 \\
\hline $0 \mathrm{H} 30$ & 8.72 & 8. 24 & 9.26 & -0.41 & 86160 & 50 & -2575 & 22 & of & -2010 & 0 & 0 & 0 & 15.00 & 245 & 220 \\
\hline $0 \mathrm{H} 40$ & B.90 & $9 .(x)$ & 9.37 & $.03 ?$ & $B B 911$ & & -2430 & 22 & or & .1875 & 0 & 0 & 0 & 15.00 & 242 & $220 \mathrm{dNV}$ \\
\hline OH SO & 9.06 & 9.14 & 9.45 & .031 & $9: 495$ & 50 & 0.2237 & 22 & OI & .1709 & 0 & 0 & 0 & 15.00 & 238 & 220 \\
\hline $1 \mathrm{HOO}$ & 9.20 & 9.27 & $\$ \$ 2$ & .0 .25 & $93 \mathrm{B63}$ & 50 & $0-1959$ & 22 & ol & -1507 & 0 & 0 & 0 & 15.00 & 234 & 220 YFC \\
\hline $1 \% 10$ & 9.33 & 9.38 & 956 & $\cdot 0.18$ & 95961 & & $-: 618$ & 22 & PD & -1655 & -14093 & -2 & -544 & 7.00 & 232 & 220 \\
\hline $1 \mathrm{H} 20$ & 9.45 & 9.49 & 9.59 & -0.10 & 97925 & & $0-i: 11$ & 22 & $P D$ & -1546 & .15124 & .4 & .1127 & 6.90 & 231 & 220 \\
\hline $1 \mathrm{H} 3 \mathrm{H}$ & 9.55 & 9.58 & 9.59 & $-0.0 ?$ & 99580 & $5 \mathrm{~F}$ & $=0$ & 22 & $\mathrm{PD}$ & .2242 & .26779 & -9 & -2153 & 11.30 & 230 & 220 \\
\hline$\because \mathrm{H}<\mathrm{O}$ & 9.63 & 9.65 & 9.57 & 0.08 & 100925 & $5 \mathrm{~F}$ & $\mathrm{~F}$ & 22 & $P D$ & .2673 & .38977 & -15 & -35.43 & 14.50 & 214 & 2200 \\
\hline $1 \% 50$ & 9.72 & 9.75 & 954 & $0.2 !$ & $102 \leq 29$ & $S F$ & $\mathrm{~F}$ & 22 & PD & .2745 & $.44 \leqslant 18$ & .23 & -4956 & 15.10 & 194 & 220 \\
\hline $2 H \infty$ & 9.82 & 9.85 & 9.49 & 0.36 & 104,77 & $5 \mathrm{~F}$ & $\mathrm{~F}$ & 22 & PD & -2731 & -48781 & .31 & .6376 & 25.40 & 171 & 2XWVFI \\
\hline $2 \mathrm{H} 10$ & 9.91 & 9.94 & 9.42 & 0.52 & 105816 & 58 & $F$ & 22 & PD & .2717 & .53303 & .40 & .7824 & 15.10 & 163 & 220 \\
\hline $2 \mathrm{H} 20$ & 10.01 & 10,03 & 934 & 0.69 & 107446 & $3 F$ & $\mathrm{~F}$ & 22 & $\mathrm{PD}$ & -2787 & -61016 & -50 & .9411 & 15.70 & 156 & 220 \\
\hline $2 \mathrm{H} 30$ & 10.10 & 10.13 & 9.25 & 0.88 & 109119 & 98 & $F$ & 22 & PD & .2758 & .65680 & .61 & -11086 & 15.60 & IS3 & 220 \\
\hline $2 \mathrm{H} 40$ & 10.20 & 10.22 & 9.14 & 1.08 & 110774 & $5 \mathrm{P}$ & 0 & 22 & PD & .2729 & -71318 & -73 & $-128 B 1$ & 15.60 & 151 & 220 \\
\hline $2 H \leq 0$ & 10.29 & 1031 & 9.02 & 1.29 & 132412 & $5 p$ & o & 22 & PD & -2715 & .77 .801 & .96 & .14826 & 15.60 & 150 & 220 \\
\hline
\end{tabular}

2. Description du programme AGRA. 
3. Tracé des niveaux - bilan du 4/12/92 (en traits continus, niveaux de la mer et de Saint Suliac prévus ; en petits pointitlés, niveau mer réel, en grands pointillés, niveau rúel du bassin).

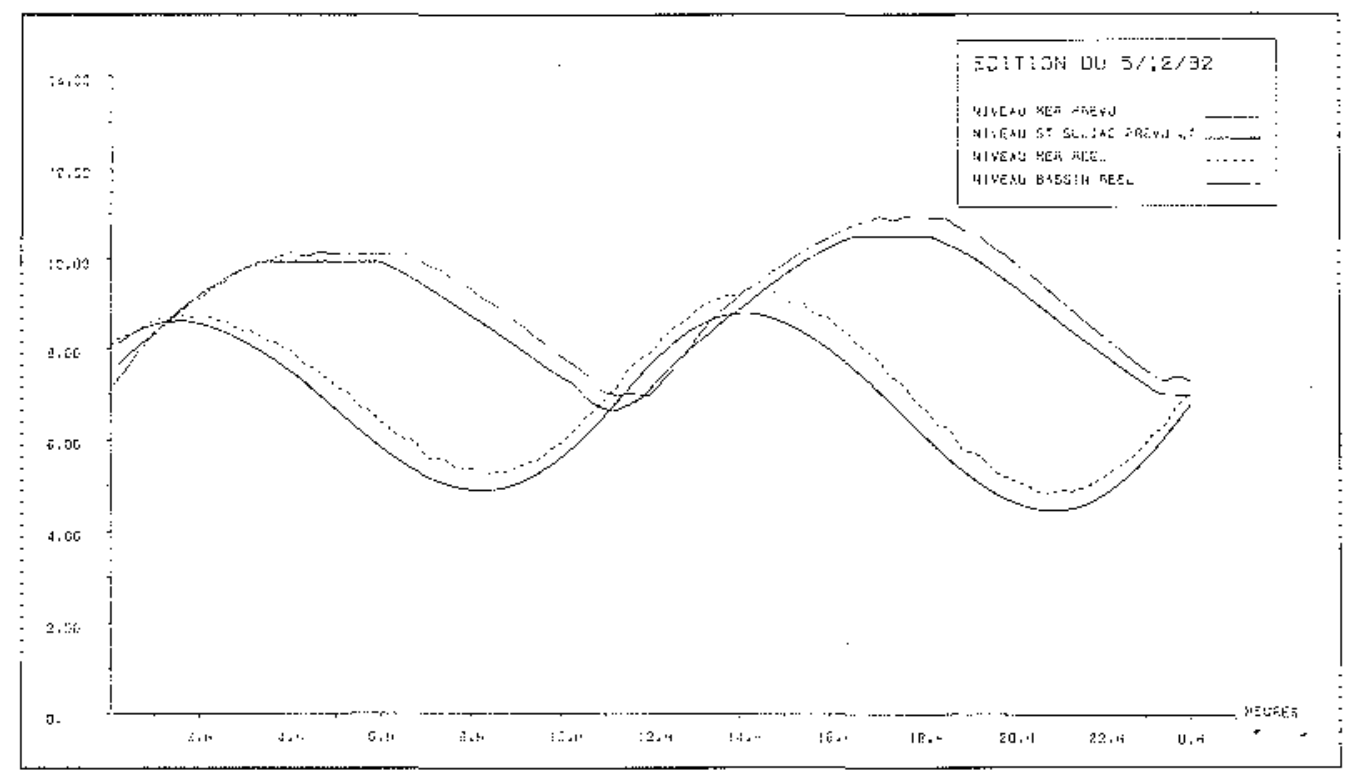

Le second rang est ainsi constitué de deux SOLAR 16-70 avec un rack partageable.

La conduite de l'usine est élaborée à partir d'informations indispensables:

- programme de conduite des différentes marches.

- tableaux de caractéristiques des groupes en fonction des différentes marches.

Le nombre très importan de ces informations impose un stockage sur une mémoire de masse constituée par un disque dur de 20 mégaoctets.

Pour s'affranchir au mieux des défaillances importantes internes au mini-calculateur (unité centrale ou disque hors service), on a choisi de doubler ces unités.

La reprise en secours d'un calculateur par l'autre est automatique et ne dépasse pas trois minutes.

Les automates peuvent détecter une absence de calculateur ECU :

— soit à cause d’une panne de liaison,

— soit par arrêt des calculateurs,

- soit pendant un basculement cálculateur.

IIs passent ators dars un ćtat dégradé provisoirc. Cet ếát dégradé deviendra définitir si les automates ne reçoivent pas un nouveau programme dans les dix minutes qui suivent.
La conduite en état dégradé pourra durer 2 heures maximum au niveau des automates.

\section{\% 4.5 Les phases du projet d'automatisation}

\section{Septembre 1984}

- passation de la commande globale de l'automatisation à Sema Metra (ex. Cerci), associé à Spic Batignolies.

Octobre 1987

- mise en service sur le site et surveillance de la conduite par le service de quart.

Elé 1988

- suppression du service de quart.

$\underline{1992}$

— demiers ajustements de l'automatisation

\section{V — CONCLUSION}

Le projet de l'automatisation de LA RANCE avait de grandes dificultés techniques à surmonter. Aujourd hui nous pouvons dire que le système mis en place est bien mâtrisé et qu'il remplit correctement l'cnsemble des fonetions exposées.

Fious observons en 1996 une moyenne d'une intervention fortuite et par semaine sur le systeme de conduite. 